\title{
Multi-spacecraft observations to study the shock extension in the inner heliosphere
}

\author{
Aline de Lucas ${ }^{1,2}$, Rainer Schwenn ${ }^{2}$, Eckart Marsch ${ }^{2}$, \\ Alisson Dal Lago ${ }^{1}$, Alicia L. Clúa de Gonzalez ${ }^{1}$, Ezequiel Echer ${ }^{1}$, \\ Walter D. Gonzalez ${ }^{1}$ and Marlos R. da Silva ${ }^{1}$ \\ 1 Instituto Nacional de Pesquisas Espaciais, \\ Av. dos Astronautas, 1758, Jardim da Granja, 12227010, São José dos Campos, São Paulo, \\ Brazil \\ email: delucas@dge.inpe.br \\ ${ }^{2}$ Max-Planck-Institut für Sonnensystemforschung - MPS, \\ Max-Planck-Str. 2, 37191, Katlenburg-Lindau, Germany \\ email: schwenn@mps.mpg.de
}

\begin{abstract}
The two Helios probes traveled at variable longitudinal and radial separations through the inner heliosphere. They collected most valuable high resolution plasma and magnetic field data for an entire solar cycle. The mission is still so successful that no other missions will collect the same kind of data in the next 20 years. One of the subjects studied after the success of the Helios mission was the identification of more than 390 shock waves driven by Interplanetary Coronal Mass Ejections (ICMEs). Combining the data from both probes, we make a statistical study for the extension of the shock waves in the interplanetary medium. For longitudinal separations of $90^{\circ}$ we found a cutoff value at this angular separation. A shock has $50 \%$ of chance to be observed by both probes and the same probability for not being observed by two spacecrafts at the same time, when the angle between them is around $90^{\circ}$. We describe the dependence of the probability for shocks to be observed by both probes with decreasing spacecraft separation. Including plasma data from the ISEE-3 and IMP-8 spacecrafts improves our statistical evaluation substantially.
\end{abstract}

Keywords. shock waves, longitudinal extension, inner heliosphere, interplanetary coronal mass ejections.

\section{Introduction}

Interplanetary shock waves are the strongest abrupt perturbation in the solar wind, playing an important role in the solar-terrestrial environment variability. They are largescale phenomena resultant of the propagation of interplanetary structures, such as ICMEs - the interplanetary counterparts of the coronal mass ejections (CMEs)(see terminology discussion by Burlaga 2001 and Russell 2001). The reason why they are formed is the fact that the relative speed between a fast stream (the ICME, in this case) and the background solar wind is often greater than the characteristic speed of the mediummagnetosonic speed. In the inner heliosphere - inside $1 A U$ - shocks driven by ICMEs are well formed, and the ICME-shock association has been observed since the first images from Solwind coronagraph were available (Sheeley et al. 1985). Sheeley et al. (1985) were the first to confirm that fast ICMEs were related to shock formation.

The purpose of this work is to study the shock longitudinal extension in the inner heliosphere using Helios, IMP-8, and ISEE-3 observations for the entire solar cycle 21. Section 2 will present the event selection and data analysis, Section 3 will discuss the results, and, finally, section 4 will summarize the conclusions. 


\section{Event Selection and Data Analysis}

Helios was a mission composed by two twin probes, Helios 1 (H1) and Helios 2 (H2), that operated at the same time from 1976 until the beginning of 1981 (Porsche 1984). Due to the long life of H1 (1974-1986), it has become possible to collect one of the most complet sets of plasma data over the time span of a full solar cycle for studying the solar wind evolution and variation into the inner heliosphere (Schwenn \& Rosenbauer 1984). Among the total set of shock waves detected by the instruments onboard Helios, 395 were classified as those driven by ICMEs. Corotating Interaction Regions (CIRs) were not included in the present work since they are normally related to shocks at distances further than $1 A U$ (Hundhausen \& Gosling 1976; Smith \& Wolfe 1976).

Each of the shocks from the full set of events was analyzed separately. Solar wind and magnetic field data from three different positions (H1, H2, and ISEE-3/or IMP-8) contributed to the comparison of the shock signatures in these reference points. This provided the opportunity to estimate the total angular distance in longitude which one could expect a shock to expand to.

By using the list of shock/ICME events studied by Sheeley et al. (1985), we had the possible flare locations associated to the limb CMEs when H1 was located close to $\pm 90^{\circ}$ from the Sun-Earth line. This enabled to correlate the shocks observed by H1 with the ones observed at Earth by IMP-8 and/or ISEE-3, once the flare location was giving further information about the possible direction the shock wave was being driven.

Among the large set of events, there were periods without observations from the solar wind and/or magnetic field instruments onboard the three missions. Sometimes gaps filled the period when the shock was expected to arrive. These cases were not included in the statistical analysis carried out in this study. Only safe events, i.e., the events with visible signatures of shocks, made part of the considered sample. However, for some of these cases with gaps, we could see a level enhancement in all solar wind parameters and magnetic field strength before and after the gap. For those cases, we saw that there was a shock, even though we could not say exactly its time of occurrence.

Another difficulty was to determine the periods when we expected IMP-8 to observe the shock arriving at Earth. The periods when IMP-8 was in the solar wind were not many, but contributed to improving the estimate, until ISEE-3 appeared in the scenario at the second half of the year 1978 with an orbit around point L1, constantly in the solar wind. Another aspect that have influenced a lot in our sample is the fact that $\mathrm{H} 2 \mathrm{did}$ not operate during the full solar cycle like H1 did. For many years we just had a constellation (a pair of probes) as an input to our statistical analysis.

The inspection is basically observational, based in the comparison among the different points of references and their observations. The left plot on Figure 1 is one example of a strong shock observed by Helios 1 at $0.952 A U$ driven by a magnetic cloud in the interplanetary medium. As it is shown in the right plot of Figure 1, the same shock was seen by $\mathrm{H} 2$ at 0.978 AU. However, the signatures of the "ejecta" are not visible on the solar wind and magnetic field parameters. Since the variation in $\vec{B}$ is very smooth and plasma $\beta$ is going down after almost one day, counting from the day of the shock, we may conclude that the probe was crossing only the shock wave and not the ICME structure itself. Near Earth, IMP-8 was the only spacecraft operating during this time (Figure 2). In this day, IMP-8 was outside the magnetospheric cavity, and the solar wind parameter profiles observed by IMP-8 were similar to the ones seen by H2. This was already expected since H2 and IMP- 8 were separated by only $9^{\circ}$. At the end of DOY 29/1977, the shock was detected by H2, and some hours later, by IMP-8. 

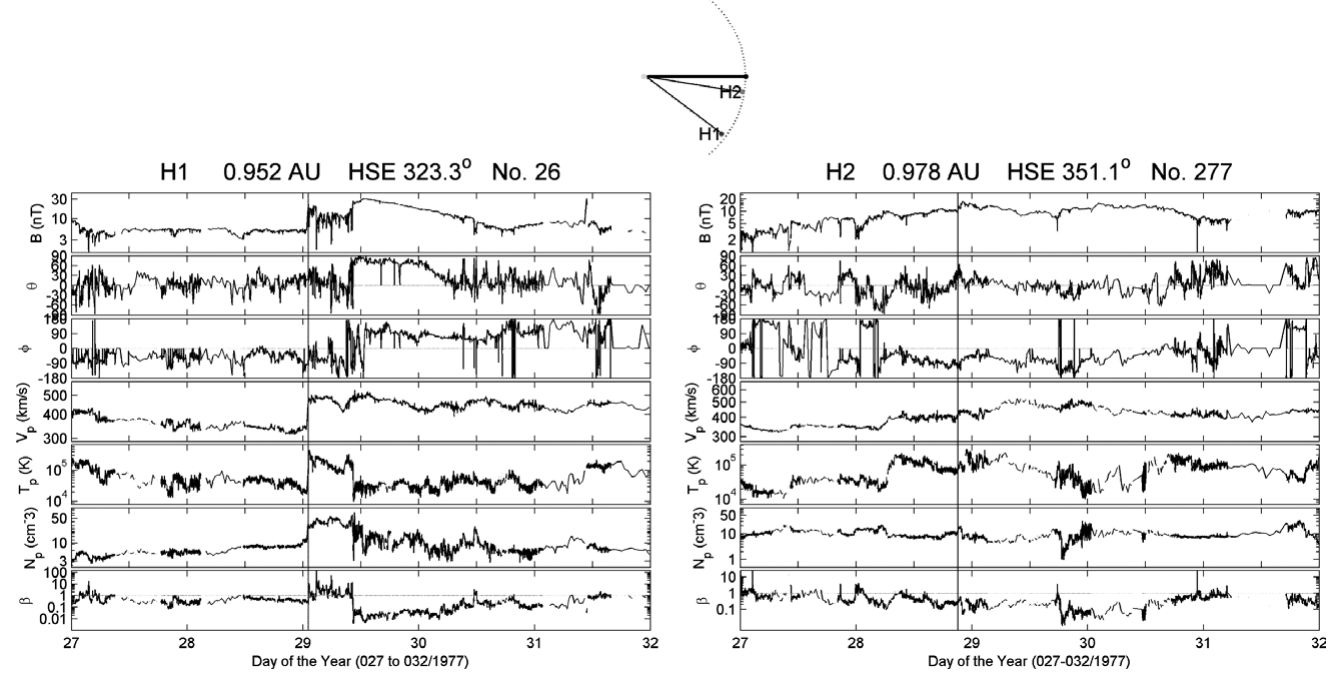

Figure 1. H1 (left plot) and H2 (right plot) magnetic field and plasma data for the shock detected on DOY 29/1977, at 01:03 UT by H1, and on DOY 28/1977, at 21:07 UT by H2. From top to bottom, one can see the magnetic field strength and angular components, followed by the solar wind proton speed, density, temperature, and the plasma beta. A magnetic cloud drives the shock, observed by $\mathrm{H} 1$ at $0.952 A U, 37^{\circ}$ away from Earth. At the top of the plot the position of the two probes $\mathrm{H} 1$ and $\mathrm{H} 2$ is shown, as well the radial distance and longitude (in the counterclockwise direction in relation to the Sun-Earth line) of H1/H2. Earth is schematically represented on the upper plot, as well the Sun, and H1 and H2 positions at the period of the shock. The Sun is the central point of the circunference sector from where the location lines of $\mathrm{H} 1$ and $\mathrm{H} 2$, for the period of the shock, originate. The thicker solid line connects the center (Sun) with Earth.

Based on the observations at the considered points we proceeded with the estimate of the shock extension in the interplanetary medium. We separated the three points of observation in three constellations of two spacecrafts each: H1 and H2, H1 and IMP8/ISEE-3, and H2 and IMP-8/ISEE-3. In Figure 1 we associated the shock occurrence on $\mathrm{H} 1$ with the one at $\mathrm{H} 2$, which means that we considered this shock as being the same in each probe. Based on this association we say that the minimum longitudinal angular distance reached by the shock was the separation between $\mathrm{H} 1$ and $\mathrm{H} 2$ - in this case the shock extended at least to $28^{\circ}$. When IMP- 8 is included in the statistical analysis a larger angle is considered: H1 and the Sun-Earth line are about $37^{\circ}$ of longitude away from each other. Again the minimum distance in longitude the shock reached was the one separating IMP- 8 and H1, since the shock was crossed by these two spacecrafts when traveling in the interplanetary medium outwardly from the Sun. The angular separation between $\mathrm{H} 2$ and IMP- 8 makes also part of the estimate once we are considering the three constelations independently.

We wanted to make sure we were seeing the same shock rather than to increase the number of cases studied without certainty. For this reason, the rate of shocks does not correspond to the total number of shocks registered during the mission. The histograms showed in the next figures are a result of two different classes: shocks observed by a pair of probes (first pannel), and shocks observed by a single probe (lower pannel).

Since our results depend on the orbit of the probes, that probably did not contribute to observe all the shocks for the period and the probes might have crossed the shocks in only one part, we might expect that there are larger angles than the ones we found. This guides us to the estimate of the margin of error. 


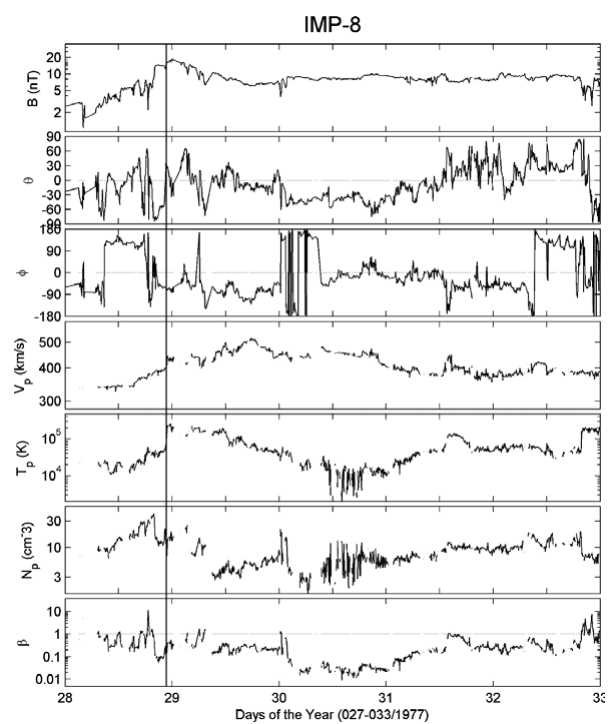

Figure 2. Interplanetary shock observed on DOY 28/1977 by IMP-8. This is the same shock previously observed by $\mathrm{H} 2$ and later on by H1. From top to bottom, one can see the profiles of the magnetic field strength and angular componentes, the solar wind protons speed, temperature, density, and plasma beta. IMP-8 was in the solar wind near Earth during the period of observation of this shock.

\section{Results}

For each case the angular separation between a pair of spacecrafts represented the minimum separation we could expect a shock to extend into the interplanetary medium. As we separated the observations according to the three different contellations, three different estimates were obtained for the whole period of observation. From the group $\mathrm{H} 1$ and H2, smaller angles separated the two probes for most of the time of operation, so our estimate was limited to the angles they formed during their orbits. From H1 and $\mathrm{H} 2$ observations we could say primarily which shocks were observed in both spacecrafts, then look for other observations near Earth - first with IMP-8 and then with ISEE-3.

When near Earth observations by IMP-8/ISEE-3 were included on the estimate, larger angles started to appear and new shock extensions were revealed. The full longitudinal range of the inner heliosphere was covered by the new points included on the observations and a new scenario for the shocks extension estimate took place. The left side of Figure 3 shows the rate of shocks for each longitudinal separation considered. In that figure, the values vary from 10 to $170^{\circ}$, and each column centered in a given $\Phi$ represents the sum of all events in the interval I $\left(\Phi \leqslant I<\left(\Phi+10^{\circ}\right)\right)$. This means that the number of cases centered in $20^{\circ}$ are a result of the number of cases in our sample where the angles were bigger than or equal to $20^{\circ}$ and smaller than $30^{\circ}$, and consecutively for the other angles in the x-axis.

As it is shown in Figure 3, there are bars that are in the right side up and others that are upside down for both the plots. The former ones correspond to those events where one of the constellations (two different points in the space) had seen the same shock. So from the different constellations separated by colors - H1 and H2 (black); H1 and IMP-8 (gray); and H2 and IMP-8 (white) -, we have the total number of cases in each angular separation considered from the set of shocks under study. And the later 
ones represent those shock waves observed by only one of the three points of reference. As it is shown in the left side of Figure 3, increasing the angular distance between two different observational points diminishes the number of events observed by each of the constellations.
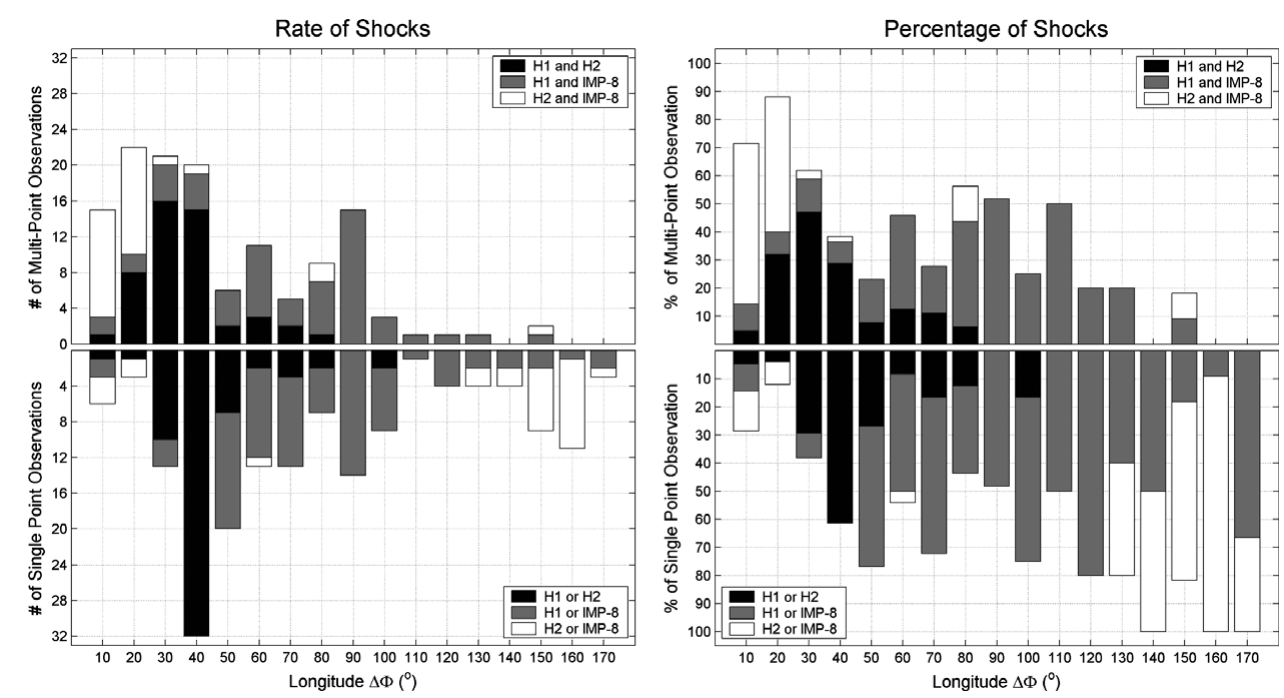

Figure 3. The figure on the left shows the number of shock waves observed from 1974 to 1985 by a pair of spacecrafts/probes (upper panel), or only by one of the spacecrafts (lower panel) as a function of the longitudinal separation $(\Delta \Phi)$ between the probes. The constellations are divided in three groups according to each pair of probes: Helios 1 and 2 (black), Helios 1 and IMP-8/ISEE-3 (gray), and Helios 2 and IMP-8/ISEE-3 (white). The figure on the right shows the same results, but as percentages.

In percentage, the distribution of our sample shows a clear trend that is illustrated in the right side of Figure 3. As we go to bigger angular separations, the percentage of shocks seen by both spacecrafts decreases, following a quasi-exponential decrease. Even though we have some special cases with large angles, like those events at $120^{\circ}, 130^{\circ}$, and $150^{\circ}$, we need to investigate them in details in order to certify that they really correspond to the same event seen in different points. According to the percentage we found in the right side of Figure 3, at $\Delta \Phi=90^{\circ}$ one has $50 \%$ of chance of seeing a shock or not seeing the same shock in two different points of observation.

The critical interval for the percentage of shocks (right side of Figure 3) was determined by using the test of proportions analysis (for details, see Kalbfleisch 1979). Figure 4 shows the error bars that represent a $95 \%$ confidence intervals for each angular separation. The estimated value is more accurate as we have a larger number of cases from the sample, like it is shown in Figure 4. A critical value at $\Delta \Phi=110^{\circ}$ is found as we have just two cases inside this angular distance.

\section{Conclusions}

We have studied shock angular extension in the inner heliosphere using observations from H1, H2, and IMP-8/ISEE-3 spacecrafts. By using a pair of these probes each time, we found that shock extension decreases as the probes angular separation increases. When a CME is observed at the solar limb, for example, there is $50 \%$ of probability of seeing 


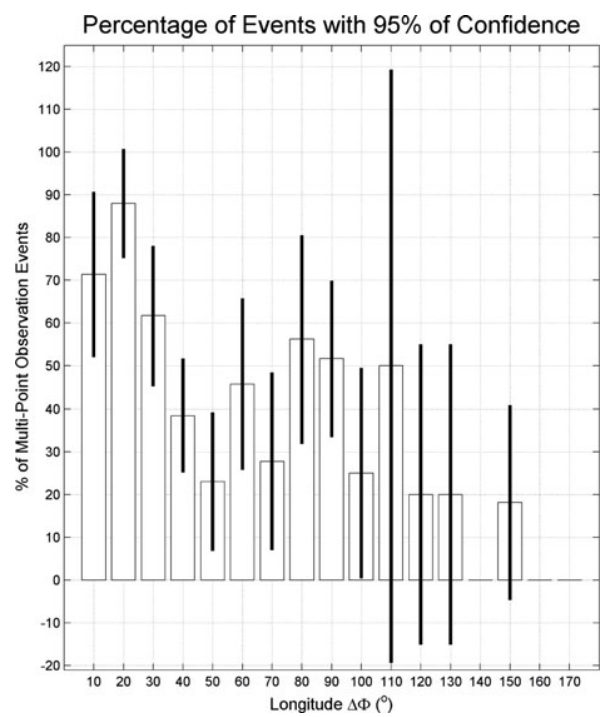

Figure 4. This is the same plot as shown before in the percentage of shocks (upper panel of Figure 3). The error margin for the percentage of shock observed by multi-points into each longitudinal separation as seen by Helios-1,2 and IMP-8/ISEE-3. As one goes further in degrees, the uncertainty for observing a shock in the angular separation $(\Delta \Phi)$ increases. Observe that in $\Delta \Phi=110^{\circ}$ the biggest error for our estimate is found. That is because only two events (left side in Figure 3) were registered for that angle: one was detected by a pair of probes, and the other, by a single probe.

the shock driven by the ICME at Earth. Further investigation is needed to evaluate those cases with large $\left(>110^{\circ}\right)$ separation.

\section{Acknowledgements}

The authors acknowledge the Instituto Nacional de Pesquisas Espaciais/INPE-MCT and the Brazilian government agency CNPQ for doctorate fellowship and projects (142012/ 2005-0, 302523/2005-7, and 472031/2007-4). The authors also thank the Max-PlanckInstitut für Sonnensystemforshung for Helios mission data, and to NSSDC for the IMP-8 and the ISEE-3 data.

\section{References}

Burlaga, L. F. 2001, Eos Trans. AGU, 82, 433

Hundhausen, A. J. \& Gosling, J. T. 1976, J. Geophys. Res., 81, 1436

Kalbfleisch, J. G. 1979, Probability and Statistical Inference II, New York: Springer-Verlag, p. 303

Porsche, H. 1984, 10 Years HELIOS, Munich: Hirmer-Verlag

Russell, C. T. 2001, Eos Trans. AGU, 82, 433

Schwenn, R. \& Rosenbauer, H. 1984, in: Porsche, H. (ed.) 10 Years HELIOS, Munich: Hirmer-Verlag, p. 66

Sheeley, Jr., N. R., Howard, R. A., Michels, D. J., Koomen, M. J., Schwenn, R., Mühlhäuser,

K. H., \& Rosenbauer, H. 1985, textitJ. Geophys. Res., 90, 163

Smith, E. J. \& Wolfe, J. H. 1976, Geophys. Res. Let., 3, 137 


\section{Discussion}

THEJAPPA: Type II radio bursts are observed as very patchy, which is interpreted in terms of ripples in the shock front. Is there any evidence for such ripple-like structure in the shock front from the two spacecraft observations, i.e., from two points of measurements from Helios?

DeLuCAS: Yes, there is. In many of the cases we studied we believe that the shock shape is represented by a ripple-line. This shape is the only explanation for these shocks we observed, when we expected the shock to arrive first at one point and it didn't. This point in general was closer to the Sun in relation to the other one with similar shock speeds and the further one observed before.

HOWARD: What was the average/most probable width of shocks that you found? I believe that one of the events on the Sheeley et al. (1985) list was a streamer blow-out event observed on one limb and the associated shock was observed when Helios was off the opposite limb, implying a longitudinal separation of more than 90 degrees from the "nose".

DELuCAS: The average width of shocks we found is mostly concentrated around 90 degrees, I would say that 90 degrees is a cutoff value for our distribution. We also have many cases observed around 30, 40, 50 degrees, but since we are limited in our angular distribution, that is the separation between the probes, we can have an even greater number of multi-point observation at other angles. 